\title{
Long-Term Outcomes of Adult Lung Transplantation Recipients: A Single-Center Experience in South Korea
}

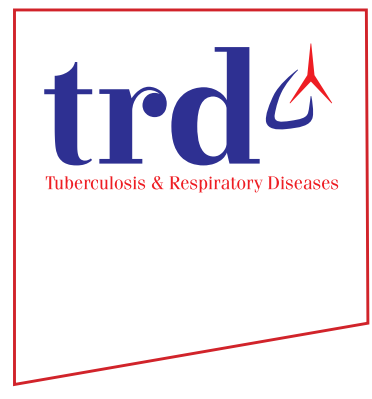

\author{
Kyung-Wook Jo, M.D., Ph.D. ${ }^{1}{ }^{(i)}$, Sang-Bum Hong, M.D., Ph.D. ${ }^{1}$, Dong Kwan Kim, M.D., Ph.D. ${ }^{2}$ \\ Sung Ho Jung, M.D., Ph.D. ${ }^{2}$, Hyeong Ryul Kim, M.D., Ph.D. ${ }^{2}$, Se Hoon Choi, M.D., Ph.D. ${ }^{2}$, \\ Geun Dong Lee, M.D., Ph.D. ${ }^{2}$, Sang-Oh Lee, M.D., Ph.D. ${ }^{3}$, Kyung-Hyun Do, M.D., Ph.D. , \\ Eun Jin Chae, M.D., Ph.D. ${ }^{4}$, In-Cheol Choi, M.D., Ph.D. ${ }^{5}$, Dae-Kee Choi, M.D., Ph.D. ${ }^{5}$, \\ In Ok Kim, M.Sc. ${ }^{6}$, Seung-Il Park, M.D., Ph.D. ${ }^{2}{ }^{\circledR}$ and Tae Sun Shim, M.D., Ph.D. ${ }^{1}{ }^{(i D}$ \\ *Author affiliations appear at the end of this article.
}

Background: Recently, the number of lung transplants in South Korea has increased. However, the long-term outcome data is limited. In this study, we aimed to investigate the long-term outcomes of adult lung transplantation recipients. Methods: Among the patients that underwent lung transplantation at a tertiary referral center in South Korea between 2008 and 2017, adults patient who underwent deceased-donor lung transplantation with available follow-up data were enrolled. Their medical records were retrospectively reviewed.

Results: Through eligibility screening, we identified 60 adult patients that underwent lung $(\mathrm{n}=51)$ or heart-lung transplantation $(n=9)$ during the observation period. Idiopathic pulmonary fibrosis $(46.7 \%, 28 / 60)$ was the most frequent cause of lung transplantation. For all the 60 patients, the median follow-up duration for post-transplantation was 2.6 years (range, 0.01-7.6). During the post-transplantation follow-up period, 19 patients (31.7\%) died at a median duration of 194 days. The survival rates were $75.5 \%, 67.6 \%$, and $61.8 \%$ at 1 year, 3 years, and 5 years, respectively. Out of the 60 patients, $8(13.3 \%)$ were diagnosed with chronic lung allograft dysfunction (CLAD), after a mean duration of $3.3 \pm 2.8$ years post-transplantation. The CLAD development rate was $0 \%, 17.7 \%$, and $25.8 \%$ at 1 year, 3 years, and 5 years, respectively. The most common newly developed post-transplantation comorbidity was the chronic kidney disease (CKD; 54.0\%), followed by diabetes mellitus (25.9\%).

Conclusion: Among the adult lung transplantation recipients at a South Korea tertiary referral center, the long-term survival rates were favorable. The proportion of patients who developed CLAD was not substantial. CKD was the most common post-transplantation comorbidity.

Keywords: Lung Transplantation; Survival; Chronic Lung Allograft Dysfunction; Chronic Kidney Disease

\footnotetext{
Address for correspondence: Seung-II Park, M.D., Ph.D.

Department of Thoracic and Cardiovascular Surgery, Asan Medical Center, University of Ulsan College of Medicine, 88 Olympic-ro 43-gil, Songpa-gu, Seoul 05505, Korea

Phone: 82-2-3010-3585, Fax: 82-2-3010-6966, E-mail: sipark@amc.seoul.kr

Address for co-correspondence: Tae Sun Shim, M.D., Ph.D.

Division of Pulmonary and Critical Care Medicine, Asan Medical Center, University of Ulsan College of Medicine, 88 Olympic-ro 43-gil, Songpa-gu, Seoul 05505, Korea

Phone: 82-2-3010-3892, Fax: 82-2-3010-6968, E-mail: shimts@amc.seoul.kr

Received: Feb. 26, 2019, Revised: May. 19, 2019, Accepted: May. 21, 2019, Published online: Aug. 31, 2019
}

@() It is identical to the Creative Commons Attribution Non-Commercial License (http://creativecommons.org/licenses/by-nc/4.0/). 


\section{Introduction}

Lung transplantation, an accepted therapeutic method for patients with end-stage lung disease, reportedly results in good functional outcomes and improved quality of life ${ }^{1}$. However, the development of chronic lung allograft dysfunction (CLAD) limits the long-term survival of these patients. Reportedly, the median survival of lung transplant recipients is only 6.0 years $^{2}$, which is comparatively lower than that of solidorgan (e.g., liver or kidney) transplant recipients ${ }^{3,4}$. Although several efforts have been made to prevent post-lung transplantation CLAD, this complication still occurs in approximately $50 \%$ of the patients at approximately 5 years post-transplantation. CLAD is attributable for approximately $30 \%$ of deaths occurring between 3 and 5 years post-transplantation ${ }^{5}$.

According to the official report of the Korean Network for Organ Sharing (KONOS), since the first lung transplantation in South Korea in 1996, the number of cumulative cases of lung transplantation had reached approximately 500 cases by $2017^{6}$. Since 2013 , more than 40 lung transplantations are performed annually in South Korea ${ }^{6}$.

Several studies have reported the short-term outcomes of lung transplantation, including airway complications, infections, and 1-year mortality ${ }^{7-9}$. However, to date, studies reporting on the long-term outcomes of lung transplantation in South Korea are limited ${ }^{10}$. We, therefore, aimed to investigate the long-term outcomes of lung transplantation recipients at one tertiary referral center, mainly focusing on (1) the survival rates, (2) the development of CLAD, and (3) the incidence of newly developed comorbidities.

\section{Materials and Methods}

\section{Study subjects}

Patients were retrospectively enrolled at Asan Medical
Center, which is a 2,700-bed referral hospital in Seoul, South Korea. In 2008, the first lung transplantation was performed at our center. Since then, until December 2017, a total of 70 patients underwent lung transplantation. After excluding (1) pediatric patients, (2) patients who simultaneously received liver transplantation, (3) a patient who underwent a livingdonor lobar lung transplantation, and (4) those whose followup data were unavailable due to the transfer to another hospital, we retrospectively evaluated the medical records of the remaining patients in November 2018 (Figure 1).

The study protocol was approved by the Institutional Review Board of the Asan Medical Center (IRB No. 2018-0414). The board waived the requirement of informed consent because of the retrospective nature of the analysis.

\section{Immunosuppressive therapy protocol}

During surgery, patients received induction immunosuppression therapy consisting of basiliximab and a high dose of intravenous methylprednisolone. Post-transplantation, the standard regimen for maintaining immunosuppression was triple therapy comprising steroids, a calcineurin inhibitor, and antimetabolites using prednisone, tacrolimus, and mycophenolate mofetil, respectively. Tacrolimus was adjusted to a target trough level of $10-15 \mathrm{ng} / \mathrm{mL}$ for the initial 6 months and 8-12 ng/mL thereafter. Mycophenolate mofetil was initiated at a daily dose of $2 \mathrm{~g}$. The dose was subsequently adjusted to achieve a trough level of $1-3 \mathrm{ng} / \mathrm{mL}$. Additionally, the dose was further adjusted based on the presence of other factors, including the patients' condition, leukopenia, or diarrhea.

\section{Infection prophylaxis strategies}

Several infection prophylaxis strategies were used ${ }^{8}$. For antiviral prophylaxis, intravenous ganciclovir was administered at a dose of $5 \mathrm{mg} / \mathrm{kg}$ every 24 hours from 1-4 weeks posttransplantation, regardless of the cytomegalovirus serostatus

70 Patients received lung or heart-lung transplantation between 2008 and 2017

\section{Excluded}

5 Pediatric patients

3 Simultaneously received liver transplantation

$\checkmark$

62 Adult patients received lung or heart-lung transplantation

\section{Excluded}

1 LDLLT

1 Transfer to other hospital
60 Adult patients received cadevaric lung or heart-lung transplantation with complete data
Figure 1. Study flow chart. LDLLT: livingdonor lobar lung transplantation. 
of recipients and donors. Thereafter, oral valganciclovir was administered at a dose of $900 \mathrm{mg}$ once daily until 6 months. For antifungal prophylaxis, voriconazole was intravenously administered at a dose of $4 \mathrm{mg} / \mathrm{kg}$ every 12 hours. Once the recipient was able to consume a normal diet, oral voriconazole was administered. The target trough level of voriconazole was $1.5-5.5 \mathrm{mg} / \mathrm{dL}$. If voriconazole was poorly tolerated or adverse effects occurred, voriconazole was replaced by itraconazole. The total duration of antifungal prophylaxis was 6 months. Lastly, oral trimethoprim/sulfamethoxazole, at a dose of $160 / 800 \mathrm{mg}$, was administered on alternate days for the recipient's lifetime to prevent pneumonia by Pneumocystis jirovecii.

\section{CLAD and prophylactic regimens}

We performed pulmonary function tests (PFTs) during outpatient visits (generally, at an interval of 1-3 months) for the diagnosis and functional grading of chronic transplant rejection. As previously defined ${ }^{11}$, CLAD was diagnosed as a persistent decline in forced expiratory volume in 1 second of at least $20 \%$ compared with the two best postoperative values, in absence of other causes. We further differentiated CLAD into bronchiolitis obliterans syndrome (BOS) or restrictive CLAD ${ }^{12,13}$. Because previous studies suggested the role of gastroesophageal reflux disease (GERD) in $\mathrm{BOS}^{14,15}$,

Table 1. Demographic characteristics, diseases, and the types of transplantation of the 60 lung transplant recipients

\begin{tabular}{|lc|}
\hline \multicolumn{1}{|c|}{ Characteristic } & \multicolumn{1}{|c|}{$\begin{array}{c}\text { Value } \\
(\mathbf{n = 6 0 )}\end{array}$} \\
\hline Age, yr & $48.1 \pm 13.6$ \\
\hline Male sex & $41(68.3)$ \\
\hline Lung transplantation indication & $28(46.7)$ \\
\hline Idiopathic pulmonary fibrosis & $7(11.7)$ \\
\hline Bronchiolitis obliterans following HSCT & $5(8.3)$ \\
\hline $\begin{array}{l}\text { Lung injury associated with the use of home } \\
\text { humidifiers }\end{array}$ & $5(8.3)$ \\
\hline Acute respiratory distress syndrome & $4(6.7)$ \\
\hline Nonspecific interstitial pneumonia & $11(18.3)$ \\
\hline Other causes* & \\
\hline Transplantation type & $51(85.0)$ \\
\hline Bilateral lung & $9(15.0)$ \\
\hline Heart-lung
\end{tabular}

Values are presented as mean $\pm \mathrm{SD}$ or number (\%).

* Other causes included acute interstitial pneumonia $(\mathrm{n}=2)$, bronchiectasis $(\mathrm{n}=2)$, persistent pulmonary hypertension following heart transplantation $(\mathrm{n}=2)$, cryptogenic organizing pneumonia $(\mathrm{n}=1)$, pneumoconiosis $(\mathrm{n}=1)$, chronic obstructive pulmonary disease $(n=1)$, pulmonary angiosarcoma $(n=1)$, and lymphangioleiomyomatosis $(\mathrm{n}=1)$.

HSCT: hematopoietic stem cell transplantation. we prophylactically prescribed a proton pump inhibitor (PPI) therapy (generally, pantoprazole $40 \mathrm{mg}$ once daily) to all patients post-transplantation for lifetime use. Additionally, as the prophylactic drug, azithromycin, is known to prevent $\mathrm{BOS}^{16}$, it was administered at a dose of $250 \mathrm{mg}$ on alternate days to all patients post-transplantation for lifetime use.

\section{Analysis for survival, completion rate of infection prophylactic regimen, and cumulative morbidities}

Initially, we evaluated survival rates using the Kaplan-Meier method at post-transplantation 1, 3, and 5 years. Further, after identifying the survivors and non-survivors at 1-year, we evaluated the recipients' data, such as demographic characteristics, the use of a mechanical ventilator and/or extracorporeal membrane oxygenation (ECMO) while awaiting transplantation, the preoperative PFT value, and the use of steroids before transplantation, between the two groups to investigate the 1-year survival-related factors. Additionally, we collected the donors' data, such as demographic characteristics, ischemic time, arterial oxygen partial pressure/fractional inspired oxygen ratio, and the cause of death, from the KONOS. Posttransplantation kidney dysfunction was evaluated the need for renal replacement therapy (RRT) after transplantation.

The completion rates of the infection prophylactic regimens and the incidence of newly developed post-transplantation comorbidities, including chronic kidney disease (CKD), diabetes mellitus, hypertension, and hyperlipidemia were investigated, after excluding patients who died within a month posttransplantation.

\section{Statistical analysis}

Continuous variables were compared using Student's t-test or the Mann-Whitney test, whereas categorical variables were compared using the chi-square or Fisher's exact test. All tests

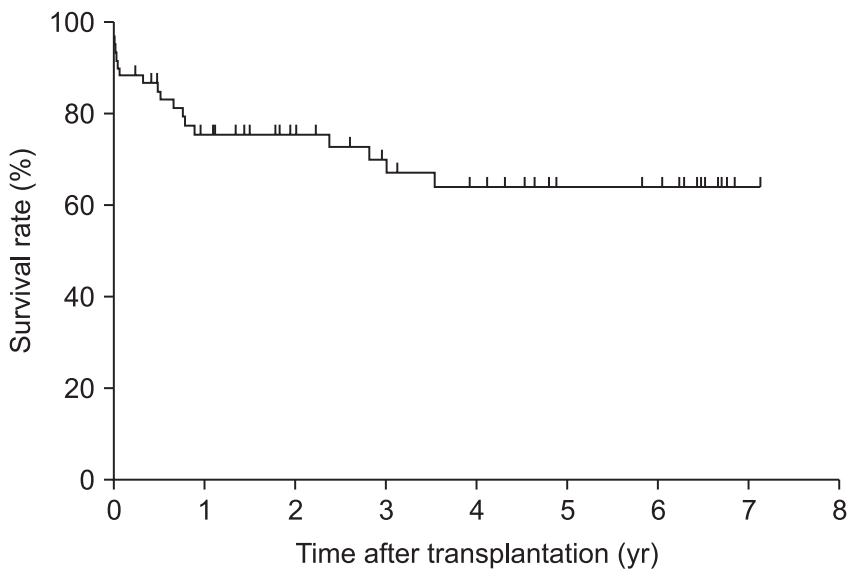

Figure 2. Kaplan-Meier survival rates of 60 patients who underwent lung transplantation. 
of significance were two-sided, and $\mathrm{p}<0.05$ was considered statistically significant. Statistical analysis was performed using SPSS version 20.0 (IBM Corp., Armonk, NY, USA).

\section{Results}

\section{Baseline characteristics}

Eligibility screening identified 60 adult patients who un- derwent lung or heart-lung transplantation (Figure 1). A total of 51 patients received bilateral lung transplants because of idiopathic pulmonary fibrosis $(\mathrm{n}=26)$, bronchiolitis obliterans following hematopoietic stem cell transplantation $(\mathrm{n}=7)$, lung injury associated with home humidifier use $(\mathrm{n}=4)^{17}$, and other diseases $(n=14)$. The remaining nine received bilateral lungheart transplants because of idiopathic pulmonary fibrosis $(n=2)$, acute respiratory distress syndrome $(n=2)$, and other diseases $(n=5)$. Patients had a mean age of $48.1 \pm 13.6$ years, and $41(68.3 \%)$ were male. Table 1 shows the age, sex, trans-

Table 2. Baseline recipient characteristics, donor characteristics, and post-transplantation kidney dysfunction of 60 patients who received lung transplants according to the 1-year survival

\begin{tabular}{|c|c|c|c|c|}
\hline Characteristic & Total $(n=60)$ & $\begin{array}{c}\text { 1-Year } \\
\text { non-survivor }(n=14)\end{array}$ & $\begin{array}{c}\text { 1-Year } \\
\text { survivor }(n=46)\end{array}$ & p-value \\
\hline Age, yr & $48.1 \pm 13.6$ & $45.4 \pm 14.1$ & $49.0 \pm 13.4$ & 0.408 \\
\hline Male sex & $41(68.3)$ & $9(64.3)$ & $32(69.6)$ & 0.749 \\
\hline Body mass index, $\mathrm{kg} / \mathrm{m}^{2}$ & $21.9 \pm 4.5$ & $22.6 \pm 5.0$ & $21.7 \pm 4.3$ & 0.521 \\
\hline Former or current smoker & $31(51.7)$ & $7(50.0)$ & $24(52.2)$ & 0.887 \\
\hline Bilateral lung transplantation & $51(85.0)$ & $12(85.7)$ & $39(84.8)$ & $>0.99$ \\
\hline Pretransplantation albumin $<3 \mathrm{~g} / \mathrm{dL}$ & $37(61.7)$ & $9(64.3)$ & $28(60.9)$ & 0.818 \\
\hline \multicolumn{5}{|l|}{ Pretransplantation PFT } \\
\hline Pre-BD FEV ${ }_{1}, \%$ pred $(\mathrm{n}=46)$ & $45.1 \pm 22.2$ & $37.7 \pm 13.6$ & $47.2 \pm 23.8$ & 0.117 \\
\hline Pre-BD FVC, \% pred $(\mathrm{n}=46)$ & $45.0 \pm 18.7$ & $39.2 \pm 14.3$ & $46.7 \pm 19.7$ & 0.196 \\
\hline $\mathrm{D}_{\mathrm{L}} \mathrm{CO}, \%$ pred $(\mathrm{n}=42)$ & $25.6 \pm 14.5$ & $30.3 \pm 17.7$ & $24.3 \pm 13.5$ & 0.367 \\
\hline 6-Minute walk distance, m (n=39) & $245.6 \pm 121.5$ & $230.8 \pm 105.6$ & $250.0 \pm 127.2$ & 0.655 \\
\hline \multicolumn{5}{|l|}{ Pretransplantation intensive care } \\
\hline Mechanical ventilator & $40(66.7)$ & $9(64.3)$ & $31(67.4)$ & 0.829 \\
\hline ECMO & $32(53.3)$ & $8(57.1)$ & $24(52.2)$ & 0.744 \\
\hline Use of prednisolone dose of $\geq 20 \mathrm{mg}$ for $\geq 2 \mathrm{wk}$ & $11(18.3)$ & $4(28.6)$ & $7(15.2)$ & 0.264 \\
\hline Active infection at the time of transplantation & $20(33.3)$ & $6(42.9)$ & $14(30.4)$ & 0.388 \\
\hline Cold ischemic time $(\mathrm{n}=59)$ & $121.3 \pm 63.5$ & $144.8 \pm 61.3$ & $125.0 \pm 70.7$ & 0.778 \\
\hline Warm ischemic time & $139.6 \pm 38.8$ & $154.0 \pm 54.5$ & $135.3 \pm 32.2$ & 0.115 \\
\hline Graft ischemic times $\geq 330$ minute $(n=59)$ & $11 / 59(18.6)$ & $4 / 13(30.7)$ & $7 / 46(15.2)$ & 0.237 \\
\hline \multicolumn{5}{|l|}{ Donor } \\
\hline Age $\geq 50 \mathrm{yr}$ & $9(15.0)$ & $4(28.6)$ & $5(10.9)$ & 0.193 \\
\hline Sex mismatch between donor and recipient & $27(45.0)$ & $6(42.9)$ & $21(45.7)$ & 0.854 \\
\hline $\mathrm{PaO}_{2} / \mathrm{FiO}_{2}$ ratio & $495.7 \pm 85.9$ & $485.1 \pm 77.2$ & $498.9 \pm 89.0$ & 0.577 \\
\hline Traumatic brain injury & $17(28.3)$ & $5(35.7)$ & $12(26.1)$ & 0.484 \\
\hline Abnormal chest X-ray findings* & $11(18.3)$ & $1(7.1)$ & $10(21.7)$ & 0.430 \\
\hline Primary graft dysfunction & $8(13.3)$ & $1(7.1)$ & $7(15.2)$ & 0.667 \\
\hline Post-transplantation RRT & $11(18.3)$ & $8(57.1)$ & $3(6.5)$ & $<0.001$ \\
\hline
\end{tabular}

Values are presented as mean \pm SD or number $(\%)$.

*Abnormal chest X-ray findings included atelectasis $(n=7)$ and pulmonary edema $(n=4)$.

PFT: pulmonary function test; $\mathrm{BD}$ : bronchodilator; $\mathrm{FEV}_{1}$ : forced expiratory volume in 1 second; \% pred: percentage of predicted value; FVC: forced vital capacity; $\mathrm{D}_{\mathrm{L}} \mathrm{CO}$ : carbon monoxide diffusing capacity; ECMO: extracorporeal membrane oxygenation; $\mathrm{PaO}_{2} / \mathrm{FiO}_{2}$ : arterial oxygen partial pressure/fractional inspired oxygen; RRT: renal replacement therapy. 
plantation type, and the indication for lung transplantation of all patients. A total of 32 patients (53.3\%) received ECMO before transplantation with a median duration of 13.5 days (range, 1.0-38.0 days).

\section{Post-transplantation survival rates and the cause(s) of death}

The median follow-up duration post-transplantation was for all 60 patients was 2.6 years (range, $0.01-7.6$ years). During the follow-up period, 19 patients (31.7\%) died in a median of 194 days (range, 3-1,297 days) after transplantation. Of these 19 patients, 14 patients died within 1-year post-transplantation. The survival rates were $75.5 \%$ at 1 year, $67.6 \%$ at 3 years, and $61.8 \%$ at 5 years. Figure 2 shows the overall survival rates of the 60 patients post-transplantation. The main cause of death within the first year after transplantation was infection (11/14 patients, $78.6 \%)$, followed by bleeding $(n=3)$. Past the first year, the causes of death of the remaining five patients included infection (n=3), BOS ( $n=1)$, and uremic encephalopathy ( $\mathrm{n}=1)$.

\section{Predictor of 1-year survival}

As shown in Table 2, the baseline characteristics of the fortysix 1-year survivors and fourteen 1-year non-survivors were comparable, except for a statistically significant difference in post-transplantation RRT between the two groups. The 1-year non-survivors received post-transplantation RRT more frequently than the survivors ( $57.1 \%$ vs. $6.5 \%$, $\mathrm{p}<0.001)$.

\section{Acute rejection and CLAD development}

A total of four patients (6.7\%) were diagnosed with acute rejection among 60 lung transplantation recipients. The median interval between operations and the diagnosis of

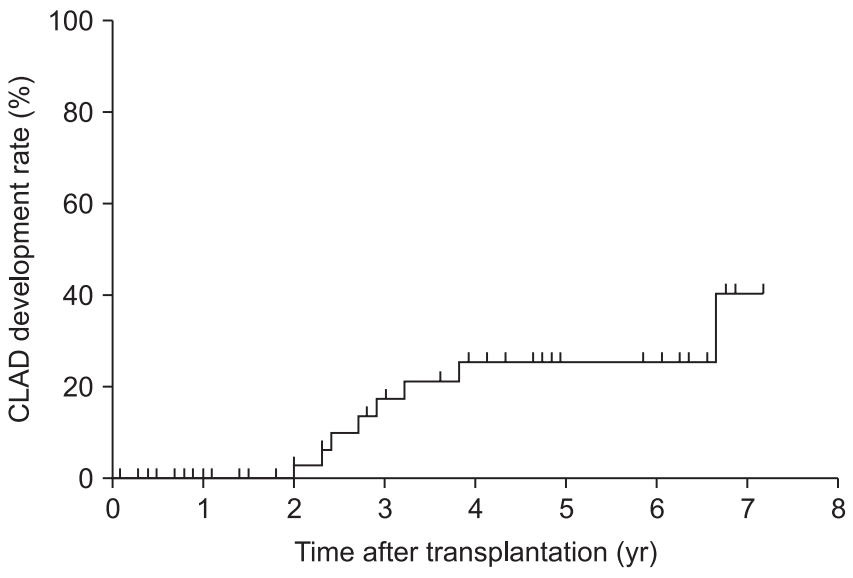

Figure 3. Kaplan-Meier estimates for chronic lung allograft dysfunction (CLAD) development of 60 patients who underwent lung transplantation. acute rejection for these four patients was 8.1 months (range, 7.7-28.9 months). Among the four patients, the diagnosis was histopathologically confirmed to be grade A2 acute cellular rejection in one patient only. The diagnosis of the remaining three patients was based on typical radiological findings combined with a dramatic response to treatment with high-dose steroids.

Further, of the 60 patients, eight (13.3\%) were diagnosed with CLAD, mostly BOS $(\mathrm{n}=7)$, after a mean duration of $3.3 \pm 2.8$ years post-transplantation. For five patients, CLAD was diagnosed at 1-3 years post-transplantation; for two patients, it was diagnosed at 3-5 years, and for one patient, it was diagnosed more than 5 years after the operation. The CLAD development rate was $0 \%$ at 1 year, $17.7 \%$ at 3 years, and $25.8 \%$ at 5 years. Figure 3 shows the Kaplan-Meier estimates of CLAD development rates.

\section{The completion rates of infection prophylactic regimens}

After excluding the six patients who died within 1-month post-transplantation, the completion rates of the infection prophylactic regimens were analyzed for the remaining 54 patients. The completion rates of the different regimens were as follows. For antiviral prophylaxis, 31 patients $(57.4 \%)$ success-

Table 3. Post-transplantation type and incidence of comorbidities and the time of diagnosis of 54 patients during the study period

\begin{tabular}{|lc|}
\hline Type and incidence of comorbidity & $\begin{array}{c}\text { Post-transplantation } \\
\text { time of diagnosis }\end{array}$ \\
\hline Chronic kidney disease $(\mathrm{n}=28,51.9 \%)$ & 21 \\
$\leq 1 \mathrm{yr}$ & 7 \\
$1-3 \mathrm{yr}$ & 0 \\
$\geq 3 \mathrm{yr}$ & \\
\hline Diabetes mellitus $(\mathrm{n}=14,25.9 \%)$ & 12 \\
$\leq 1 \mathrm{yr}$ & 2 \\
$1-3 \mathrm{yr}$ & 0 \\
$\geq 3 \mathrm{yr}$ & \\
\hline Hyperlipidemia $(\mathrm{n}=11,20.4 \%)$ & 7 \\
$\leq 1 \mathrm{yr}$ & 3 \\
$1-3 \mathrm{yr}$ & 1 \\
$\geq 3 \mathrm{yr}$ & \\
\hline Systemic hypertension $(\mathrm{n}=6,11.1 \%)^{*}$ & 4 \\
$\leq 1 \mathrm{yr}$ & 2 \\
$1-3 \mathrm{yr}$ & 0 \\
$\geq 3 \mathrm{yr}$ & \\
\hline
\end{tabular}

*If patients received antihypertensive medication because of combined chronic kidney disease, we did not consider it as newly diagnosed systemic hypertension. 
fully completed a ganciclovir/valganciclovir regimen. A total of 18 patients (33.3\%) had to discontinue this treatment because of cytopenia; the remaining five patients discontinued the regimen because of death $(n=3)$, cost $(n=1)$, and vomiting $(n=1)$. For antifungal prophylaxis, 38 patients (70.4\%) completed a prophylactic voriconazole regimen without interruption. The most common cause of discontinuation in the remaining patients was visual hallucinations. Oral trimethoprim/sulfamethoxazole was continuously administered to 48 patients (88.9\%) without the occurrence of any adverse effects.

\section{Incidence of newly developed comorbidities}

After excluding the six patients who died within 1-month post-transplantation, the incidence of newly developed comorbidities was investigated for the remaining 54 patients. Among these, various comorbidities developed during a median follow-up time of 2.8 years (range, $0.1-7.6$ years). As shown in Table 3, the most common comorbidity was CKD $(\mathrm{n}=28,51.9 \%)$, which mostly occurred within 1 year after transplantation. Of the 28 patients with CKD, maintenance immunosuppressive regimen was switched to a combination of mammalian target of rapamycin (mTOR) inhibitors and other immunosuppressive agents such as cyclosporine in five patients. The second most common comorbidity was diabetes mellitus ( $\mathrm{n}=14,25.9 \%$ ). The Kaplan-Meier estimates for development rates of newly developed comorbidities are shown in Supplementary Figures S1-4.

\section{Discussion}

Although the number of lung transplants has steadily escalated in recent years in South Korea, the literature concerning long-term outcomes, such as survival, rate of CLAD development, and newly developed comorbidities, is limited. Our most important findings were that the long-term survival rates of our subjects were favorable, the proportion of patients who developed CLAD was not substantial, and a substantial portion of patients developed comorbidities, such as CKD or diabetes mellitus, post-transplantation.

We observed a favorable outcome in terms of post-transplantation survival rates. The 1-, 3-, and 5-year survival rates of our study subjects were comparable with those recently reported $(82 \%, 69 \%$, and $59 \%$, respectively) by the International Society for Heart and Lung Transplantation and are remarkably better than those reported by KONOS $(60.3 \%, 50.6 \%$, and $47.9 \%$, respectively) in $2016^{2,18}$. The main difference between our subjects and those of the KONOS registry is that our subjects were consecutive patients from a single center. It was not until the past two consecutive years that our institute performed 10-20 lung transplantation cases per year. Additionally, lung transplantation experience of our center is limited to only 10 years. However, our outcomes are comparable with those of the international registry. We believe that several factors contributed to this favorable outcome: (1) well-defined pre-established protocols in place, (2) prudent selection of deceased donors and recipients, (3) good inter- and multidisciplinary collaboration among the various departments, and (4) the effectiveness of the prophylactic regimens in preventing infection and CLAD. Notably, we have never performed single-lung transplantation, which has a worse survival rate than bilateral transplantation ${ }^{19,20}$.

Among our subjects, the only significant predictor of 1-year survival was post-transplantation RRT. This finding is consistent with the findings of the previous studies ${ }^{9,21,22}$. One study showed that post-transplantation RRT was associated with increased in-hospital and 1-year mortality ${ }^{22}$. George et al. ${ }^{21}$ reported that the requirement of post-transplantation RRT was strongly associated with short- and long-term mortality in 12,108 patients from the United Network for Organ Sharing database. In South Korea, a study from another tertiary referral center reported that needing a post-transplantation RRT was an independent perioperative risk factor for 1-year mortality ${ }^{9}$. The need for RRT post-transplantation could be considered a state of critical illness ${ }^{9}$. That is, the patients who develop multiorgan dysfunction post-transplantation are less likely to tolerate medications, showing nephrotoxicity, perioperative hemodynamic instability, and eventually requiring RRT $^{21}$.

The survival benefits of ECMO use as a bridge for lung transplantation have long been debated. It has been reported that lung transplant recipients undergoing ECMO have increased short- and long-term mortality ${ }^{23}$, particularly those who receive a transplant after waiting for $>14$ days $\mathrm{s}^{24}$. However, some recent reports showed that a combination of mechanical ventilation and ECMO is a viable bridging strategy, leading to acceptable patient outcomes ${ }^{25}$. We believe that our subjects were extremely critical wherein approximately $50 \%$ of them received ECMO while awaiting transplantation. In contrast, of the $>9,000$ patients from the United Network for Organ Sharing (UNOS) database between 2005 and 2011, only approximately $1 \%$ received ECMO support ${ }^{26}$. The reason for the high percentage of patients receiving ECMO in our study is related to the Korean lung allocation score criteria, which states that only patients who are connected to a ventilator or ECMO belong to the category status 0 (the most emergent state).

CLAD is the leading cause of death at 1 -year post-transplantation ${ }^{13}$. The International Society for Heart and Lung Transplantation (ISLHT) registry reported that approximately $50 \%$ of patients developed BOS within 5 years posttransplantation. In contrast, the incidence of CLAD was lower in our lung transplantation recipients than those in the ISLHT registry. We thought that several factors contributed to lower CLAD development in our subjects. First, immediately after the transplantation, we routinely prescribed PPI to prevent chronic rejection in all patients. GERD induces rejection by 
triggering a non-allogenic injury to the transplanted lung, and reflux-mediated aspiration is a risk factor for $\mathrm{BOS}^{14,15}$. We also routinely administered azithromycin to all patients. This is because one double-blind, placebo-controlled trial showed that prophylactic azithromycin significantly improved BOSfree survival ${ }^{16}$. The long-term completion rate of these drugs is satisfactory: $>90 \%$ of our study subjects completed PPI and azithromycin without the occurrence of adverse effects. Second, vast evidence exists of the association of microbial, fungal, and viral colonization/infection with BOS development ${ }^{27}$. Therefore, our institute's protocol includes the routine prophylactic use of voriconazole and ganciclovir/valganciclovir, which may also help prevent BOS development. However, approximately $50 \%$ of our subjects could not complete ganciclovir/valganciclovir prophylaxis.

In our study, we reported the occurrence of various newly developed comorbidities, such as CKD and diabetes mellitus; CKD was the most common comorbidity in our subjects (51.9\%), mostly occurring within 1-year post-transplantation. The incidence of CKD development (51.9\%) during a median follow-up of 2.8 years is comparable with the incidence of CKD development (55\%) reported in an international registry ${ }^{28}$. CKD development after transplantation is generally attributed to chronic calcineurin inhibitor nephrotoxicity ${ }^{29}$. Tacrolimus is also known to be associated with the development of comorbidities such as post-transplantation diabetes mellitus, hypertension, and hyperlipidemia ${ }^{30}$. For patients with kidney dysfunction, adding an mTOR inhibitor and reducing the calcineurin inhibitor dose has shown to improve kidney function $^{31-33}$. Of the 28 patients with CKD in our study, some patients were switched to the administration of sirolimus and cyclosporine to prevent CKD progression.

A limitation of our study was that it was a single-center retrospective review with a limited number of patients. Thus, our study population is probably not completely representative of the lung transplant recipient population in South Korea. Further, our relatively short follow-up period could not adequately reveal the long-term adverse effects.

In conclusion, for adult lung transplant recipients in a tertiary referral center in South Korea, the long-term survival rates are favorable and the rate of CLAD development was not substantial. CKD was the most common newly developed comorbidity post-transplantation, and it occurred mostly within 1-year post-transplantation.

\section{*Author Affiliations}

${ }^{1}$ Division of Pulmonology and Critical Care Medicine, Departments of ${ }^{2}$ Thoracic and Cardiovascular Surgery, ${ }^{3}$ Infectious Diseases, ${ }^{4}$ Radiology, and ${ }^{5}$ Anesthesiology and Pain Medicine, Asan Medical Center, University of Ulsan College of Medicine, Seoul, ${ }^{6}$ Organ Transplantation Center,
Asan Medical Center, Seoul, Korea

\section{Authors' Contributions}

Conceptualization: Jo KW, Park SI, Shim TS. Methodology: Jo KW, Park SI, Shim TS. Formal analysis: Jo KW, Shim TS. Data curation: Jo KW, Hong SB, Kim DK, Jung SH, Kim HR, Choi SH, Lee GD, Lee SO, Do KH, Chae EJ, Choi IC, Choi DK, Kim IO. Validation: Jo KW, Hong SB. Investigation: Jo KW, Park SI, Shim TS. Writing - original draft preparation: Jo KW, Park SI, Shim TS. Writing - review and editing: Jo KW, Park SI, Shim TS. Approval of final manuscript: all authors.

\section{Conflicts of Interest}

No potential conflict of interest relevant to this article was reported.

\section{Funding}

No funding to declare.

\section{Supplementary Material}

Supplementary material can be found in the journal homepage (http://www.e-trd.org).

Supplementary Figure S1. Kaplan-Meier estimates for chronic kidney disease development of 54 patients who underwent lung transplantation.

Supplementary Figure S2. Kaplan-Meier estimates for diabetes mellitus development of 54 patients who underwent lung transplantation.

Supplementary Figure S3. Kaplan-Meier estimates for hyperlipidemia development of 54 patients who underwent lung transplantation.

Supplementary Figure S4. Kaplan-Meier estimates for hypertension development of 54 patients who underwent lung transplantation.

\section{References}

1. Rutherford RM, Fisher AJ, Hilton C, Forty J, Hasan A, Gould FK, et al. Functional status and quality of life in patients surviving 10 years after lung transplantation. Am J Transplant 2005;5:1099-104.

2. Chambers DC, Yusen RD, Cherikh WS, Goldfarb SB, Kucheryavaya AY, Khusch K, et al. The Registry of the International Society for Heart and Lung Transplantation: Thirty-fourth 
Adult Lung And Heart-Lung Transplantation Report-2017; Focus Theme: Allograft ischemic time. J Heart Lung Transplant 2017;36:1047-59.

3. Morris PJ. Transplantation: a medical miracle of the 20th century. N Engl J Med 2004;351:2678-80.

4. Waki K. UNOS Liver Registry: ten year survivals. Clin Transpl 2006:29-39.

5. Verleden GM, Vos R, Vanaudenaerde B, Dupont L, Yserbyt J, Van Raemdonck D, et al. Current views on chronic rejection after lung transplantation. Transpl Int 2015;28:1131-9.

6. Korean Network for Organ Sharing. National data for organ transplantation [Internet]. Seoul: Korean Network for Organ Sharing; 2019 [cited 2019 May 7]. Available from: https:// www.konos.go.kr/konosis/index.jsp.

7. Cho EN, Haam SJ, Kim SY, Chang YS, Paik HC. Anastomotic airway complications after lung transplantation. Yonsei Med J 2015;56:1372-8.

8. Yun JH, Lee SO, Jo KW, Choi SH, Lee J, Chae EJ, et al. Infections after lung transplantation: time of occurrence, sites, and microbiologic etiologies. Korean J Intern Med 2015;30:50614.

9. Lee SH, Park MS, Song JH, Kim YS, Lee JG, Paik HC, et al. Perioperative factors associated with 1-year mortality after lung transplantation: a single-center experience in Korea. J Thorac Dis 2017;9:4006-16.

10. Jeong YH, Choi S, Park SI, Kim DK; Asan Medical Center Lung Transplantation Team. Clinical outcomes of lung transplantation: experience at Asan Medical Center. Korean J Thorac Cardiovasc Surg 2018;51:22-8.

11. Verleden GM, Raghu G, Meyer KC, Glanville AR, Corris P. A new classification system for chronic lung allograft dysfunction. J Heart Lung Transplant 2014;33:127-33.

12. Verleden SE, Ruttens D, Vandermeulen E, Bellon H, Van Raemdonck DE, Dupont LJ, et al. Restrictive chronic lung allograft dysfunction: where are we now? J Heart Lung Transplant 2015;34:625-30.

13. Vos R, Verleden SE, Verleden GM. Chronic lung allograft dysfunction: evolving practice. Curr Opin Organ Transplant 2015;20:483-91.

14. Patti MG, Vela MF, Odell DD, Richter JE, Fisichella PM, Vaezi MF. The intersection of GERD, aspiration, and lung transplantation. J Laparoendosc Adv Surg Tech A 2016;26:501-5.

15. Basseri B, Conklin JL, Pimentel M, Tabrizi R, Phillips EH, Simsir SA, et al. Esophageal motor dysfunction and gastroesophageal reflux are prevalent in lung transplant candidates. Ann Thorac Surg 2010;90:1630-6.

16. Vos R, Vanaudenaerde BM, Verleden SE, De Vleeschauwer SI, Willems-Widyastuti A, Van Raemdonck DE, et al. A randomised controlled trial of azithromycin to prevent chronic rejection after lung transplantation. Eur Respir J 2011;37:16472.

17. Kim WY, Hong SB. Humidifier disinfectant-associated lung injury: six years after the tragic event. Tuberc Respir Dis 2017;
80:351-7.

18. Korean Network for Organ Sharing. National data for organ transplantation [Internet]. Seoul: Korean Network for Organ Sharing; 2019 [cited 2019 May 7]. Available from: https:// www.konos.go.kr/konosis/common/bizlogic.jsp?fwdUrl=/ sub4/sub04_06_01_list.jsp\&boardid=22\&event=search2\&svc =web.wm.law.

19. Schaffer JM, Singh SK, Reitz BA, Zamanian RT, Mallidi HR. Single- vs double-lung transplantation in patients with chronic obstructive pulmonary disease and idiopathic pulmonary fibrosis since the implementation of lung allocation based on medical need. JAMA 2015;313:936-48.

20. Puri V, Patterson GA, Meyers BF. Single versus bilateral lung transplantation: do guidelines exist? Thorac Surg Clin 2015; 25:47-54.

21. George TJ, Arnaoutakis GJ, Beaty CA, Pipeling MR, Merlo CA, Conte JV, et al. Acute kidney injury increases mortality after lung transplantation. Ann Thorac Surg 2012;94:185-92.

22. Arnaoutakis GJ, George TJ, Robinson CW, Gibbs KW, Orens JB, Merlo CA, et al. Severe acute kidney injury according to the RIFLE (risk, injury, failure, loss, end stage) criteria affects mortality in lung transplantation. J Heart Lung Transplant 2011;30:1161-8.

23. Jayarajan SN, Taghavi S, Komaroff E, Brann S, Horai T, Cordova $\mathrm{F}$, et al. Impact of extracorporeal membrane oxygenation or mechanical ventilation as bridge to combined heart-lung transplantation on short-term and long-term survival. Transplantation 2014;97:111-5.

24. Crotti S, Iotti GA, Lissoni A, Belliato M, Zanierato M, Chierichetti $\mathrm{M}$, et al. Organ allocation waiting time during extracorporeal bridge to lung transplant affects outcomes. Chest 2013; 144:1018-25.

25. Hayanga AJ, Du AL, Joubert K, Tuft M, Baird R, Pilewski J, et al. Mechanical ventilation and extracorporeal membrane oxygenation as a bridging strategy to lung transplantation: significant gains in survival. Am J Transplant 2018;18:125-35.

26. George TJ, Beaty CA, Kilic A, Shah PD, Merlo CA, Shah AS. Outcomes and temporal trends among high-risk patients after lung transplantation in the United States. J Heart Lung Transplant 2012;31:1182-91.

27. Verleden SE, Sacreas A, Vos R, Vanaudenaerde BM, Verleden GM. Advances in understanding bronchiolitis obliterans after lung transplantation. Chest 2016;150:219-25.

28. Yusen RD, Christie JD, Edwards LB, Kucheryavaya AY, Benden C, Dipchand AI, et al. The Registry of the International Society for Heart and Lung Transplantation: Thirtieth Adult Lung and Heart-Lung Transplant Report, 2013; focus theme: age. J Heart Lung Transplant 2013;32:965-78.

29. Bloom RD, Doyle AM. Kidney disease after heart and lung transplantation. Am J Transplant 2006;6:671-9.

30. Floreth T, Bhorade SM, Ahya VN. Conventional and novel approaches to immunosuppression. Clin Chest Med 2011;32: 265-77. 
31. Scheffert JL, Raza K. Immunosuppression in lung transplantation. J Thorac Dis 2014;6:1039-53.

32. Shitrit D, Rahamimov R, Gidon S, Bakal I, Bargil-Shitrit A, Milton S, et al. Use of sirolimus and low-dose calcineurin inhibitor in lung transplant recipients with renal impairment: results of a controlled pilot study. Kidney Int 2005;67:1471-5.
33. Gullestad L, Iversen M, Mortensen SA, Eiskjaer H, Riise GC, Mared L, et al. Everolimus with reduced calcineurin inhibitor in thoracic transplant recipients with renal dysfunction: a multicenter, randomized trial. Transplantation 2010;89:86472 . 